\title{
Minimal Log Gravity
}

\author{
Gaston Giribet ${ }^{1,2} \quad$ Yerko Vásquez ${ }^{3}$
}

September 19, 2018

\author{
${ }^{1}$ Departamento de Física, Universidad de Buenos Aires FCEN-UBA and IFIBA-CONICET, \\ Ciudad Universitaria, Pabellón I, 1428, Buenos Aires, Argentina. \\ ${ }^{2}$ Instituto de Física, Pontificia Universidad Católica de Valparaíso, \\ Casilla 4950, Valparaíso, Chile. \\ ${ }^{3}$ Departamento de Física, Universidad de la Serena, \\ Avenida Cisternas 1200, La Serena, Chile.
}

\begin{abstract}
Minimal Massive Gravity (MMG) is an extension of three-dimensional Topologically Massive Gravity that, when formulated about Anti-de Sitter space, accomplishes to solve the tension between bulk and boundary unitarity that other models in three dimensions suffer from. We study this theory at the chiral point, i.e. at the point of the parameter space where one of the central charges of the dual conformal field theory vanishes. We investigate the non-linear regime of the theory, meaning that we study exact solutions to the MMG field equations that are not Einstein manifolds. We exhibit a large class of solutions of this type, which behave asymptotically in different manners. In particular, we find analytic solutions that represent two-parameter deformations of extremal Bañados-Teitelboim-Zanelli (BTZ) black holes. These geometries behave asymptotically as solutions of the so-called Log Gravity, and, despite the weakened falling-off close to the boundary, they have finite mass and finite angular momentum, which we compute. We also find time-dependent deformations of BTZ that obey Brown-Henneaux asymptotic boundary conditions. The existence of such solutions shows that Birkhoff theorem does not hold in MMG at the chiral point. Other peculiar features of the theory at the chiral point, such as the degeneracy it exhibits in the decoupling limit, are discussed.
\end{abstract}

\section{Introduction}

Minimal Massive Gravity (MMG), introduced in Ref. [1, is an extension of three-dimensional Topologically Massive Gravity (TMG) which, when formulated about Anti-de Sitter space (AdS), accomplishes solving the tension between bulk and boundary unitarity. As TMG, MMG about $\mathrm{AdS}_{3}$ propagates a single local degree of freedom [2. However, in contrast to what happens in other models of three-dimensional gravity such as TMG or New Massive Gravity (NMG) [3, in $M M G$ the $\mathrm{AdS}_{3}$ graviton excitation happens to have positive energy for the same values of coupling constants for which the central charges of the dual conformal field theory (CFT) turn 
out to be positive. Therefore, MMG seems to solves this bulk-boundary unitarity puzzle in an ingenious way [1, 4].

Having a minimal model of three-dimensional gravity that allows for positive graviton excitations and, at the same time, positive central charges in the dual theory, a natural question arises as to what happens with the graviton excitation at the so-called chiral point, i.e. at the point of the parameter space where one of the two central charges of the dual $\mathrm{CFT}_{2}$ vanishes. This question is natural as the physics of the graviton excitation at the chiral point was the main point in the discussion about the consistency of the so-called Chiral Gravity; see [5, 6, 7, 8, 9,

Being parity-odd theories, both TMG and MMG in $\mathrm{AdS}_{3}$ have an asymptotically symmetry algebra generated by two copies of Virasoro algebra with different central charges, $c_{ \pm}$. The difference between these central charges, $c_{+}-c_{-}$, controls the diffeomorphism anomaly in the dual $\mathrm{CFT}_{2}$. There is a point (or a curve) in the parameter space where one of these central charges (say $c_{-}$) vanishes. It is commonly believed that at that point, and provided suitable boundary conditions are imposed, the boundary theory becomes a chiral $\mathrm{CFT}_{2}$. At the chiral point, the bulk theory also exhibits peculiar features, like the appearance of new solutions with different boundary conditions. Depending on the asymptotic boundary conditions considered, the local degrees of freedom of the bulk theory can vary. For instance, in the case of TMG at the chiral point, there exist two different models that coexist, each of them exhibiting substantially different features: One such theory is Chiral Gravity, originally proposed by Li, Song and Strominger in Ref. [5], which is defined by considering TMG on $\mathrm{AdS}_{3}$ at the point $c_{-}=0$ and imposing Brown-Henneaux boundary conditions [10]. The other theory is the so-called Log Gravity [9], which is defined by considering the same action with the same values of the coupling constants, but demanding a weakened version of the asymptotic boundary conditions originally proposed by Grumiller and Johansson in Ref. [7]. While the former theory is conjectured to be dual to a chiral $\mathrm{CFT}_{2}$, there is evidence suggesting that the latter is dual to a non-unitary (logarithmic) $\mathrm{CFT}_{2}$.

Unlike Chiral Gravity, Log Gravity exhibits a propagating degree of freedom (a bulk graviton). In Ref. [7], the energy of this graviton was computed and shown to be negative (for values of the couplings for which $c_{+}$was positive). Besides, in Ref. [8] it was shown that such graviton causes a linear instability in the theory, making necessary to go to second order in perturbation theory to capture its actual asymptotic behavior [9]. An important piece of information to confirm that a theory with a propagating graviton with weakened falling-off at the chiral point $c_{-}=0$ actually existed was given in Ref. [11, where it was proven that exact solutions obeying the boundary conditions of [7] but not obeying those of [10] actually exist. This permitted bringing the discussion between Chiral Gravity versus Log Gravity beyond the linear and next-to-linear level. One of the results of this paper is to extend this analysis to the case of MMG. We will show that MMG at the chiral point $\left(c_{-}=0\right)$ admits exact solutions that are asymptotically $\mathrm{AdS}_{3}$ in the sense proposed in Ref. [7] but fail to obey Brown-Henneaux boundary conditions. Some of the solutions we exhibit correspond to a two-parameter deformation of the extremal Bañados-Teitelboim-Zanelli (BTZ) black holes [12, with a deformation that may behave asymptotically either as the graviton of Log Gravity or as a Brown-Henneaux solution. We compute the conserved charges of these MMG solutions and show that, despite the weakened asymptotics, the charges are finite. We also consider the question as to whether exact solutions exist which, while obeying the stronger Brown-Henneaux asymptotics, happen not to be solutions of three-dimensional General Relativity. The (non-)existence of such solutions at 
the chiral point was an important ingredient in the discussion of [9] about the contributions to the Chiral Gravity partition function. Solutions of this sort for the case of TMG were subsequently found in Ref. [13], and here we show how they can be generalized and extended to MMG. In particular, this leads us to show that in this theory Birkhoff theorem does not hold, in the sense that there exist circularly symmetric vacuum solutions that are time-dependent. We find such time-dependent solutions explicitly.

The paper is organized as follows: In Section 2, we briefly introduce MMG theory in $\mathrm{AdS}_{3}$. In

Section 3, we study the theory at the chiral point at non-linear level. That is, we study analytic solutions of the theory that are not the simple extensions of General Relativity solutions. In particular, we present a two-parametric deformation of the extremal BTZ solution which has nonvanishing mass and angular momentum at the chiral point. These solutions are explicit examples of solutions that behaves asymptotically as Log Gravity solutions. We also find analytic timedependent deformations of the extremal BTZ black hole, showing that Birkhoff theorem does not hold in this model. Nevertheless, we show that these time-dependent excitations carry zero conserved charges. We also find a much more general set of solutions, with different asymptotics. In Section 4, we present our conclusions.

\section{Minimal Log Gravity}

\subsection{Minimal Massive Gravity}

MMG is an extension of TMG. It can be conveniently described in the so-called first order formalism, i.e. in terms of the vielbein one-form $e^{a}=e_{\mu}^{a} d x^{\mu}$ and the spin connection one-form $\omega^{a b}=\omega_{\mu}^{a b} d x^{\mu}$ (where Latin indices refer to the tangent space). First, let us recall that TMG can also be described in such a way by first considering the Einstein-Hilbert action with cosmological constant and then adding to it the exotic Chern-Simons term for $\omega^{a b}$ plus a term that couples a Lagrange multiplier $\lambda_{a}$ to the torsion two-form $T^{a}=d e^{a}+\omega_{b}^{a} \wedge e^{b}$; see for instance [14. Then, the MMG is defined by augmenting the TMG action written in the first order formalism by also including a term that is quadratic in $\lambda^{a}$. In this way, the variation of the action with respect to $\omega^{a b}$ produces an algebraic equation for $\lambda^{a}$, while the equation of motion associated to the variation with respect to $e^{a}$ involves its covariant derivative $d \lambda^{a}+\omega_{b}^{a} \wedge \lambda^{b}$; see also [15]. This yields a set of third order field equations that defines MMG. In the second order formalism, the equations of motion of MMG read

$$
\sigma G_{\mu \nu}+\Lambda g_{\mu \nu}+\frac{1}{\mu} C_{\mu \nu}=-\frac{\gamma}{\mu^{2}} J_{\mu \nu}
$$

where $G_{\mu \nu}$ is the Einstein tensor, $G_{\mu \nu} \equiv R_{\mu \nu}-\frac{1}{2} R g_{\mu \nu}$, and $C_{\mu \nu}$ is the Cotton tensor

$$
C_{\mu \nu}=\frac{\epsilon_{\mu}{ }^{\rho \sigma}}{\sqrt{-g}} \nabla_{\rho} S_{\sigma \nu}
$$

with $S_{\mu \nu}$ being the Schouten tensor, $S_{\mu \nu} \equiv R_{\mu \nu}-\frac{1}{4} R g_{\mu \nu}$, and where the tensor $J_{\mu \nu}$ is defined also in terms of $S_{\mu \nu}$ as follows

$$
J_{\mu \nu}=\frac{1}{2} \frac{\epsilon_{\mu}{ }^{\rho \sigma}}{\sqrt{-g}} \frac{\epsilon_{\nu}^{\tau \eta}}{\sqrt{-g}} S_{\rho \tau} S_{\sigma \eta} .
$$


In (2.1), $\Lambda$ is the cosmological constant, $\mu$ is a coupling constant with mass dimension one, and $\gamma$ is an arbitrary dimensionless constant. $\sigma$ stands for a coefficient that can be set to $\sigma= \pm 1$ by rescaling the other coupling constants.

One can verify that (2.3) can be written as

$$
J_{\mu \nu}=R_{\mu}{ }^{\rho} R_{\rho \nu}-\frac{3}{4} R R_{\mu \nu}-\frac{1}{2} g_{\mu \nu}\left(R^{\rho \sigma} R_{\rho \sigma}-\frac{5}{8} R^{2}\right) .
$$

Since $J_{\mu \nu}$ is of second order in the metric, the Lanczos-Lovelock theorem implies that it can not be covariantly conserved; that is, the quantity $\nabla_{\mu} J_{\nu}^{\mu}$ can not vanish identically. In fact, one can show that

$$
\nabla_{\mu} J_{\nu}^{\mu}=\frac{\epsilon_{\nu}^{\rho \sigma}}{\sqrt{-g}} S_{\rho}^{\eta} C_{\eta \sigma}
$$

and it does not vanish identically. However, $\nabla_{\mu} J_{\nu}^{\mu}=0$ actually holds on-shell, once equations of motion (2.1) are imposed. The trace of (2.1) reduces to

$$
\sigma R-6 \Lambda+\frac{\gamma}{\mu^{2}}\left(R_{\mu \nu} R^{\mu \nu}-\frac{3}{8} R^{2}\right)=0
$$

which coincides with the trace of NMG field equations [3] for a graviton mass $m=\mu / \sqrt{\gamma}$.

The fact that covariant derivative (2.5) does not vanish identically immediately suggests that the theory would present at least two problems. First, a problem will emerge when trying to couple the theory to matter. This issue was addressed in Ref. [16], where the kind of matter content to which MMG can actually be coupled consistently was investigated. A second problem is related to the computation of conserved charges. Conservation of the field equations is a crucial step in the derivation of the conserved charge formulae. This issue was recently studied in Ref. [17, where a method to compute conserved charges in MMG was proposed. This consists of an extension of the Abbott-Deser-Tekin (ADT) method, adapted to the peculiar case of MMG. In this paper, we do not need to face any of these two problems because, on the one hand, we will consider the theory (2.1) in vacuum and, on the other hand, we will employ the definition of conserved charges worked out in [17] which, as we will see, in our case also leads to consistent results.

\subsection{Minimal Massive Gravity in $\mathrm{AdS}_{3}$}

We are interested in the theory about $\mathrm{AdS}_{3}$ space, which is obviously a solution of (2.1). Assuming $R_{\mu \nu}=-\left(2 / \ell^{2}\right) g_{\mu \nu}$ in (2.6), one finds

$$
\ell^{2}=\frac{1}{2 \Lambda}\left(-\sigma \pm \sqrt{1-\gamma \Lambda / \mu^{2}}\right)
$$

which gives an expression for the effective cosmological constant $\Lambda_{\text {eff }} \equiv-1 / \ell^{2}$ in terms of the coupling constants of the theory (provided $\mu^{2} \geq \gamma \Lambda$ ).

The theory about $\mathrm{AdS}_{3}$ is conjectured to be dual to a two-dimensional conformal field theory with central charges given by 1

$$
c_{ \pm}=\frac{3 \ell}{2 G}\left(\sigma+\frac{\gamma}{2 \mu^{2} \ell^{2}} \pm \frac{1}{\mu \ell}\right)
$$

\footnotetext{
${ }^{1}$ Here, we will adopt the notation of Ref. [17].
} 
with a normalization that leads to the Brown-Henneaux result $c_{ \pm}=3 \ell /(2 G)$ valid for General Relativity [10] in the limit $\mu \rightarrow \infty$, and reproduces the expression for TMG when $\gamma=0$.

The central charge $c_{ \pm}$vanishes at the point

$$
\mu^{2} \ell^{2} \sigma \pm \mu \ell+\gamma / 2=0
$$

of the parameter space. In the case $\gamma=0$ this reduces to the TMG chiral point $\mu \ell= \pm 1$. As for the case of TMG, we will refer to the point $c_{-}=0$ as the chiral point of the theory. The theory has also another interesting point at $\mu^{2}=\gamma \Lambda$, but we will not study it here.

\section{Non-linear solutions}

\section{$3.1 p p$-wave solutions in $\mathrm{AdS}_{3}$}

The first class of exact solutions we will consider is $p p$-wave solutions in $\mathrm{AdS}_{3}$ space, also known as AdS-waves. For TMG these solutions were studied in Ref. [18]. In MMG, AdS-waves solutions were also studied recently [19], and here we review them because they are useful solutions to gain intuition about the theory.

Let us start with the $\mathrm{AdS}_{3}$ metric written in Poincaré coordinates; namely

$$
d s_{0}^{2}=\frac{\ell^{2}}{z^{2}}\left(-2 d x^{+} d x^{-}+d z^{2}\right),
$$

where $z \in \mathbb{R}_{\geq 0}$. In these coordinates, the boundary of the space is located at $z=0$. These coordinates, with $x^{ \pm} \in \mathbb{R}$, describe the Poincaré patch of $\mathrm{AdS}_{3}$ space.

For MMG to admit the AdS vacuum (3.1), the $\mathrm{AdS}_{3}$ radius $\ell$, the cosmological constant $\Lambda$, and the couplings parameters $\mu$ and $\gamma$ must obey (2.7) for $\ell^{2}>0$. The scale of the cosmological constant, $\ell_{\Lambda} \equiv|\Lambda|^{-1 / 2}$ and the $\mathrm{AdS}_{3}$ radius $\ell$ coincide when $\mu \rightarrow \infty$ or $\gamma=0$.

The metrics of $\mathrm{AdS}_{3}$-waves can be written as

$$
d s^{2}=\frac{\ell^{2}}{z^{2}}\left(-F\left(x^{+}, z\right)\left(d x^{+}\right)^{2}-2 d x^{+} d x^{-}+d z^{2}\right) .
$$

These metrics are conformally-related to that of the $p p$-waves.

The $\mathrm{AdS}_{3}$-wave solutions (3.2) describe exact gravitational waves propagating on an $\mathrm{AdS}_{3}$ background; therefore, we have to consider the value (2.7) for the effective cosmological constant. With this choice, the equation of motion reads

$$
\frac{1}{4 \ell^{4} \mu^{3} z}\left(-\left(\gamma \mu+2 \ell^{2} \mu^{3} \sigma\right) \ell^{2} \frac{\partial F}{\partial z}+\left(\gamma \mu+2 \ell^{2} \mu^{3} \sigma\right) \ell^{2} z \frac{\partial^{2} F}{\partial z^{2}}-2 \ell^{3} \mu^{2} z^{2} \frac{\partial^{3} F}{\partial z^{3}}\right)=0 .
$$

Considering a solution of the form $F \propto z^{\alpha}$, the characteristic polynomial reads

$$
\alpha(\alpha-2)\left(\ell^{2} \gamma \mu-2 \ell^{3}(\alpha-1) \mu^{2}+2 \ell^{4} \mu^{3} \sigma\right)=0,
$$

and generic solution for the wave profile is

$$
F\left(x^{+}, z\right)=F_{+}\left(x^{+}\right)(z / \ell)^{1+\ell \mu \sigma+\frac{\gamma}{2 \ell \mu}}
$$


where the function $F_{+}\left(x^{+}\right)$only depends on the coordinate $x^{+}$. The solutions $\alpha=0$ and $\alpha=2$ can be eliminated by coordinate transformations.

In addition to the power law behavior (3.5), new logarithmic modes appear for some values of the parameters: For the special point of the parameter space $\mu^{2} \ell^{2} \sigma+\mu \ell+\gamma / 2=0$, such a logarithmic solution exists and is given by

$$
F\left(x^{+}, z\right)=F_{+}\left(x^{+}\right) \log (z) .
$$

Analogously, for the special point $\mu^{2} \ell^{2} \sigma-\mu \ell+\gamma / 2=0$, the logarithmic solution is given by

$$
F\left(x^{+}, z\right)=F_{+}\left(x^{+}\right)\left(z^{2} \log (z)-z^{2} / 2\right) .
$$

In these logarithmic solutions we have discarded the constant term and the quadratic term in the variable $z$, because, as said, can be eliminated by coordinate transformations.

The appearance of logarithmic $\mathrm{AdS}_{3}$-waves solutions suggests that much more general solutions with such near-boundary behavior exist in the theory at the chiral point. In the next subsections we will see that this is indeed the case. For instance, such as one can act on $\mathrm{AdS}_{3}$ space with global transformations and generate in this way interesting causal structures, like black holes, there exists a rich variety of solutions that are locally equivalent to $\mathrm{AdS}_{3}$-waves discussed above and exhibit interesting properties.

\subsection{Ansatz for non-linear deformation of $\mathrm{AdS}_{3}$}

Let us begin by considering the vacuum metric (3.1) and a deformation of the form

$$
d s^{2}=d s_{0}^{2}+H_{-}(t, r)\left(d x^{-}\right)^{2}+H_{+}(t, r)\left(d x^{+}\right)^{2},
$$

where $H_{ \pm}(t, r)$ are two functions that may depend on time and the radial direction (with coordinates $r \equiv \sqrt{2} \ell^{2} / z$ and $\left.x^{ \pm} \equiv t \pm \ell \phi\right)$. Notice that here we are preserving the circular symmetry but allowing for non-stationary solutions. In fact, we will see that time-dependent solutions for $H_{ \pm}(t, r)$ exist.

In terms of coordinates $r=\sqrt{2} \ell^{2} / z, t=\left(x^{+}+x^{-}\right) / 2$ and $\phi=\left(x^{+}-x^{-}\right) /(2 \ell)$, metric (3.8) takes the form

$$
d s^{2}=-\frac{r^{2}}{\ell^{2}} d t^{2}+\frac{\ell^{2}}{r^{2}} d r^{2}+r^{2} d \phi^{2}+H_{-}(t, r)(d t-\ell d \phi)^{2}+H_{+}(t, r)(d t+\ell d \phi)^{2} .
$$

This turns out to be a particularly convenient ansatz to solve field equations (2.1). Inserting the form (3.9) in the MMG equations of motion, one obtains coupled third order differential equation for functions $H_{ \pm}(t, r)$ that, despite the complexity of the higher-curvature terms, in some cases can be solved analytically. Here, we are interested in the chiral point $c_{-}=0$, namely in the case when $\mu^{2} \ell^{2} \sigma-\mu \ell+\gamma / 2=0$. At this point, a solution to the MMG equations of motion with the form

$$
H_{-}(r)=2 k \log (r)+k_{0}
$$

arises; with $H_{+}$being zero and with $k$ and $k_{0}$ being two arbitrary constants. It is easy to check that this solution, which is the simplest case of a much more general class we will studied below, behaves asymptotically (i.e. at large $r$ ) as the Log Gravity excitations [7, 9], representing a 
non-linear realization of the theory. Besides, also at the point $c_{-}=0$, one finds time-dependent solutions of the form

$$
H_{-}(r, t)=\tilde{k} t-\frac{\tilde{k}^{2} k_{\gamma}}{r^{4}}+k_{0},
$$

with $H_{+}=0$, where $\tilde{k}, k_{0}$ and $k_{\gamma}$ are constants. While $\tilde{k}$ and $k_{0}$ are arbitrary, constant $k_{\gamma}$ is determined in terms of $\gamma$ and $\ell$ in a precise way we will describe below. Deformation (3.11) provides a time-dependent solution of the MMG equations of motion. It is worthwhile noticing that, in contrast to (3.10), (3.11) behaves asymptotically respecting Brown-Henneaux boundary conditions. It can be shown that, despite being time-dependent, this solution carries vanishing mass; see (3.32) below.

Also at $c_{-}=0$, one finds solutions that deform the $\mathrm{AdS}_{3}$ asymptotic in a much more drastic way. For instance, one finds

$$
H_{+}(r)=2 \hat{k} r^{2} \log (r)+k_{0},
$$

with $H_{-}=0$ and with $\hat{k}$ being an arbitrary constant. Of course, this latter solution has its mirror image $H_{-}(r)=2 \hat{k} r^{2} \log (r)+k_{0}$ with $H_{+}=0$ at $c_{+}=0$ (i.e. when $\ell^{2} \mu^{2} \sigma+\ell \mu+\gamma / 2=0$.)

In the next subsections, we will see how these solutions can be generalized and, in particular, lead to a two-parameter deformation of the extremal BTZ black hole.

\subsection{Logarithmic deformations of BTZ solution}

Let us begin by considering the extremal BTZ black hole solution; namely

$$
d s_{\mathrm{eBTZ}}^{2}=-N^{2}(r) d t^{2}+\frac{d r^{2}}{N^{2}(r)}+r^{2}\left(N_{\phi}(r) d t-d \phi\right)^{2},
$$

with the metric functions

$$
N^{2}(r)=\frac{r^{2}}{\ell^{2}}-M+\frac{M^{2} \ell^{2}}{4 r^{2}}, \quad N_{\phi}=\frac{M \ell}{2 r^{2}},
$$

and with $M$ being an integration constant. This solution, which for $M>0$ represents a maximally rotating black hole in three dimensions, solves Einstein equations with negative $\Lambda$ in three dimensions [12] and, then, it also solves the MMG field equations provided $\ell^{2}>0$. The black hole horizon is located at $r_{H}=\sqrt{M \ell^{2} / 2}$. At the chiral point $\ell^{2} \mu^{2} \sigma-\ell \mu+\gamma / 2=0$, solution (3.13)-(3.14) has vanishing mass and vanishing angular momentum [17].

Now, consider a deformation of the form

$$
d s^{2}=d s_{\mathrm{eBTZ}}^{2}+N_{k}^{2}(r)(d t-\ell d \phi)^{2} .
$$

The case $M=0$ in (3.13)-(3.15) corresponds to $H_{-}(r)=N_{k}^{2}(r), H_{+}=0$ in (3.9). Remarkably, at $\ell^{2} \mu^{2} \sigma-\ell \mu+\gamma / 2=0$ the MMG field equations admit the following configuration as an exact solution for arbitrary $M$,

$$
N_{k}^{2}(r)=k \log \left(\left(r^{2}-M \ell^{2} / 2\right) / r_{0}^{2}\right),
$$

where $k$ and $r_{0}$ are two arbitrary constants. Notice that (3.16) reduces to (3.10) in the case $M=0$. Asymptotically, (3.16) also behaves as a Log Gravity solution, as it damps-off slower than 
Brown-Henneaux configurations [10] while obeys the Grumiller-Johansson weakened boundary conditions [7]. More precisely, solution (3.16) behaves at large $r$ as follows

$$
\begin{aligned}
& g_{t t} \simeq \frac{r^{2}}{\ell^{2}}+\mathcal{O}(\log (r)), \quad g_{r r} \simeq \frac{\ell^{2}}{r^{2}}+\mathcal{O}\left(r^{-4}\right), \\
& g_{t \phi} \simeq \mathcal{O}(\log (r)), \quad g_{\phi \phi} \simeq r^{2}+\mathcal{O}(\log (r)),
\end{aligned}
$$

where $\mathcal{O}\left(r^{-n}\right)$ stands for functions of $t$ and $\phi$ whose large $r$ behavior damps-off faster or equal than $1 / r^{n}$. The presence of $\mathcal{O}(\log (r))$ is a typical feature that three- and higher-dimensional higher-curvature gravity theories exhibit at critical points of the moduli space.

Solution (3.13)-(3.16) represents a one-parameter deformation of the extremal BTZ solution. As we will see in the next subsection, it carries non-zero conserved charges, which are given in terms of the integration constant $k$. The case $k=0$ reduces to the extremal BTZ solution. Even for $k \neq 0$, all the curvature scalars associated to this solution are constants and independent of $k$; nevertheless, the solution exhibits a pathology at $r=\sqrt{M \ell^{2} / 2}$, where the horizon of the case $k=0$ is located. At that radius, the effective potential of geodesics becomes infinite, tending either to $+\infty$ or $-\infty$ depending on the sign of $k$. For $k \neq 0$, components $g_{t t}, g_{t \phi}$ and $g_{\phi \phi}$ of the metric blow up at the radius $r=\sqrt{M \ell^{2} / 2}$, where the would be horizon is located. The analysis of the geodesic equations shows that angular velocity tends to infinity as the particles approach that radius.

The solution (3.13)-(3.16), which has isometry group $S O(2) \times \mathbb{R}$, is not locally $\mathrm{AdS}_{3}$ if $k \neq 0$. In fact, it is not even conformally flat.

At the point $\ell^{2} \mu^{2} \sigma+\ell \mu+\gamma / 2=0$ we obtain also a deformation of BTZ with the form

$$
\widehat{N}_{k}^{2}(r)=k\left(r^{2}-M \ell^{2} / 2\right) \log \left(\left(r^{2}-M \ell^{2} / 2\right) / r_{0}^{2}\right) .
$$

This represents a much more drastic deformation of the $\mathrm{AdS}_{3}$ asymptotics. However, close to the horizon it behaves much better that (3.16), as (3.19) vanishes in the limit $r \rightarrow \sqrt{M \ell^{2} / 2}$.

\subsection{Conserved charges}

Now, let us compute the conserved charges of the solutions described above. We will employ the method proposed by Tekin in Ref. [17, which provides a definition of conserved charges in MMG. To avoid repetition, we will not review the details of the method of [17] here; instead, we will refer to the original paper and to the seminal works [21, 22]. Nevertheless, to facilitate the discussion, we will work with the same notation as in [17.

Let us denote by $g_{\mu \nu}$ the spacetime metric and by $\bar{g}_{\mu \nu}$ the background metric, respect to which the charges will be computed. These metrics have the same asymptotic Killing symmetries, generated by $\bar{\xi}$, and are related by $g_{\mu \nu} \equiv \bar{g}_{\mu \nu}+h_{\mu \nu}$. Then, the charges are given by the following formula,

$$
Q^{\mu}(\bar{\xi})=\frac{1}{2 \pi G} \oint d l_{i}\left(\left(\sigma+\frac{\gamma}{2 \ell^{2} \mu^{2}}\right) q_{E}^{\mu i}(\bar{\xi})+\frac{1}{2 \mu} q_{E}^{\mu i}(\bar{\Xi})+\frac{1}{2 \mu} q_{C}^{\mu i}(\bar{\xi})\right)
$$

with the integral being evaluated on a circle at spatial infinity. Functions $q_{E}^{\mu i}(\bar{\xi}), q_{E}^{\mu i}(\bar{\Xi})$ and $q_{C}^{\mu i}(\bar{\xi})$ are defined in [17] (see also Ref. [20]), and read 


$$
\begin{aligned}
q_{E}^{\mu i}(\bar{\xi})= & \sqrt{-\bar{g}}\left(\bar{\xi}_{\nu} \bar{\nabla}^{\mu} h^{i \nu}-\bar{\xi}_{\nu} \bar{\nabla}^{i} h^{\mu \nu}+\bar{\xi}^{\mu} \bar{\nabla}^{i} h-\bar{\xi}^{i} \bar{\nabla}^{\mu} h+h^{\mu \nu} \bar{\nabla}^{i} \bar{\xi}_{\nu}-\right. \\
& \left.h^{i \nu} \bar{\nabla}^{\mu} \bar{\xi}_{\nu}+\bar{\xi}^{i} \bar{\nabla}_{\nu} h^{\mu \nu}-\bar{\xi}^{\mu} \bar{\nabla}_{\nu} h^{i \nu}+h \bar{\nabla}^{\mu} \bar{\xi}^{i}\right), \\
q_{C}^{\mu i}(\bar{\xi})= & \epsilon^{\mu i \beta} \mathcal{G}_{\nu \beta} \bar{\xi}^{\nu}+\epsilon^{\nu i \beta} \mathcal{G}^{\mu}{ }_{\beta} \bar{\xi}_{\nu}+\epsilon^{\mu \nu \beta} \mathcal{G}^{i}{ }_{\beta} \bar{\xi}_{\nu},
\end{aligned}
$$

where $\bar{\Xi}^{\beta}=\epsilon^{\alpha \nu \beta} \bar{\nabla}_{\alpha} \bar{\xi}_{\nu} / \sqrt{-\bar{g}}$ and where $\mathcal{G}_{\mu \nu}$ is the linearized cosmological Einstein tensor

$$
\mathcal{G}_{\mu \nu}=R_{\mu \nu}^{L}-\frac{1}{2} \bar{g}_{\mu \nu} R^{L}+\frac{2}{\ell^{2}} h_{\mu \nu}
$$

with the linearized Ricci tensor $R_{\mu \nu}^{L}$ given by

$$
\begin{aligned}
R_{\mu \nu}^{L} & =\frac{1}{2}\left(-\bar{\square} h_{\mu \nu}-\bar{\nabla}_{\mu} \bar{\nabla}_{\nu} h+\bar{\nabla}^{\sigma} \bar{\nabla}_{\nu} h_{\sigma \mu}+\bar{\nabla}^{\sigma} \bar{\nabla}_{\mu} h_{\sigma \nu}\right), \\
R^{L} & =R_{\mu \nu}^{L} \bar{g}^{\mu \nu}+\frac{2}{\ell^{2}} h=-\bar{\square} h+\bar{\nabla}_{\mu} \bar{\nabla}_{\nu} \bar{h}^{\mu \nu}+\frac{2}{\ell^{2}} h
\end{aligned}
$$

with $h=\bar{g}^{\mu \nu} h_{\mu \nu}$. Notice that all contractions and raising and lowering indices must be done with the background metric $\bar{g}_{\mu \nu}$.

For a timelike Killing vector $\bar{\xi}^{\mu}=(-1,0,0)$, (3.20) corresponds to the energy, while for the spacelike Killing vector $\bar{\xi}^{\mu}=(0,0,1)$, it corresponds to the angular momentum. Then, choosing the BTZ black hole with $M=0$ and $J=0$ as the background metric $\bar{g}_{\mu \nu}$, we obtain the mass and the angular momentum corresponding to the Killing vectors $\bar{\xi}^{\mu}=-\partial_{t}$ and $\bar{\xi}^{\mu}=\partial_{\phi}$, respectively. For the solution given by equations (3.15)-(3.16) at the chiral point $\ell^{2} \mu^{2} \sigma-\ell \mu+\gamma / 2=0$, the conserved charges are given by

$$
\mathcal{M}=\frac{2 k}{\mu \ell G}, \quad \mathcal{J}=\frac{2 k}{\mu G} .
$$

As expected, in the limit $\gamma=0$ (3.26) agrees with the result for TMG if the appropriate normalization of the action is considered; cf. Eq. (3.51) of Ref. 14]; see also [11.

Then, what we have found here is an exact one-parameter deformation of the extremal BTZ black hole that behaves asymptotically as a Log Gravity solution and, besides, carries nonvanishing mass and angular momentum. In the following subsections we will generalize this solution further.

\subsection{Time-dependent deformations}

Now, let us consider other exact solutions, which represent different type of deformations of BTZ. Consider again the ansatz

$$
d s^{2}=-N^{2}(r) d t^{2}+\frac{d r^{2}}{N^{2}(r)}+r^{2}\left(N_{\phi}(r) d t-d \phi\right)^{2}+\tilde{N}_{\tilde{k}}^{2}(t, r)(d t-\ell d \phi)^{2},
$$

where now the deformation function depends on time. One can verify that at the chiral point $\ell^{2} \mu^{2} \sigma-\ell \mu+\gamma / 2=0$ the field equations are solved for

$$
\tilde{N}_{\tilde{k}}^{2}(t, r)=\tilde{k} t-\frac{\tilde{k}^{2} \ell^{6}}{N\left(r^{2}-M \ell^{2} / 2\right)^{2}},
$$


where

$$
N=\frac{96}{5-4 \ell \mu \sigma}=\frac{96}{1+2 \gamma /(\ell \mu)} .
$$

The case $M=0$ of (3.28) corresponds to (3.11) with $\tilde{k}=k / \ell^{2}, k_{\gamma}=\ell^{6} / N$. This timedependent solution generalizes one of the non-linear solutions of TMG found in Ref. [13]. Notice that, in fact, in the case $\ell \mu \sigma=1$ (i.e. $\gamma=0$ ) the particular case $M=0$ of (3.11) reduces to Eq. (3.22) of Ref. [13, for which $N=96$. It also generalizes solutions of TMG coupled to NMG studied in Ref. 23] to the case $M \geq 0$.

Solution (3.27)-(3.29) presents a large $r$ behavior consistent with the expansion

$$
\begin{aligned}
& g_{t t} \simeq \frac{r^{2}}{\ell^{2}}+\mathcal{O}\left(r^{0}\right), \quad g_{r r} \simeq \frac{\ell^{2}}{r^{2}}+\mathcal{O}\left(r^{-4}\right), \\
& g_{t \phi} \simeq \mathcal{O}\left(r^{0}\right), \quad g_{\phi \phi} \simeq r^{2}+\mathcal{O}\left(r^{0}\right),
\end{aligned}
$$

so that it satisfies the Brown-Henneaux boundary conditions [10].

The existence of this type of solutions in the case of TMG was relevant for the discussion about the contribution to the partition function of Chiral Gravity [9]. This is because one of the assumptions in [9] was the non-existence of solutions obeying Brown-Henneaux boundary conditions and not being Einstein manifolds. The discovery of such a solution in [13] proved that there exist non-Einstein contributions. Here, we have shown that also in MMG in $\mathrm{AdS}_{3}$ at the chiral point, solutions exist that obey Brown-Henneaux asymptotic boundary conditions not being Einstein manifolds.

On the other hand, the existence of time-dependent circularly symmetric solution (3.27)(3.29) manifestly shows that in this theory Birkhoff theorem does not hold. A natural question is what are the conserved charges associated to this time-dependent deformation of extremal BTZ. Notice that, despite being time-dependent, the linear dependence on time appears in a next-to-leading term in the large $r$ expansion. One can use the method of [17] described in the previous subsection and verify that the mass and angular momentum associated to (3.27)-(3.29) actually vanish; namely

$$
\mathcal{M}=0, \quad \mathcal{J}=0 .
$$

This raises the question as to whether non-Einstein solutions obeying Brown-Henneaux conditions and having non-vanishing conserved charges actually exist.

There is also another interesting point, which is where $N$ in 3.29 diverges; that is, when $\ell \mu \sigma=-\gamma \sigma=5 / 4$. There, a solution appears which corresponds to replacing $\tilde{N}_{\tilde{k}}^{2}(t, r)$ in (3.28)

by $\tilde{N}_{\tilde{k}}^{2}(t, r)=k_{2} r^{2}+\tilde{k} t+k_{0}+N_{k}^{2}(r)$, with $N_{k}^{2}(r)$ given by (3.16). Let us discuss this type of solutions with more free parameters in the next subsection.

\subsection{Two-parameter deformations and generalizations}

Remarkably, if we add solutions (3.15) and (3.27), a new solution to the field equations at the chiral point $\ell^{2} \mu^{2} \sigma-\ell \mu+\gamma / 2=0$ is obtained; namely

$$
H_{-}(t, r)=N_{k}^{2}(r)+\tilde{N}_{\tilde{k}}^{2}(t, r),
$$

with $N_{k}^{2}(r)$ and $\tilde{N}_{\tilde{k}}^{2}(t, r)$ defined in equations (3.16) and (3.28) also solves the MMG field equations for arbitrary $k$ and $\tilde{k}$. We obtain in this way a two-parameter deformation of extremal 
BTZ. The conserved charges of this general solution can also be computed and shown to yield (3.26).

Besides, one can explore a much more general ansatz for $H_{ \pm}$, including dependence on the angular coordinate $\phi$, and still find analytic solutions. For instance, perturbing the $M=0$ solution at the chiral point $\mu^{2} \ell^{2} \sigma-\mu \ell+\gamma / 2=0$, one finds that

$$
d s^{2}=-\frac{r^{2}}{\ell^{2}} d t^{2}+\frac{\ell^{2}}{r^{2}} d r^{2}+\frac{r^{2}}{\ell^{2}} d x^{2}+H_{-}(t, r, x)(d t-d x)^{2},
$$

with $H_{+}=0$ and $H_{-}(t, r, x=\ell \phi)$ solves the field equations for the following expansion

$$
H_{-}(t, r, x)=h_{-}^{(2)}(t, x) r^{2}+h_{-}^{(0)}(t, x)+h_{-}^{(0+)} \log (r)+h_{-}^{(-4)} r^{-4} .
$$

where $h_{-}^{(n)}(t, x)$ stand for functions of $t$ and $x$ that organize the different terms of order $\mathcal{O}\left(r^{n}\right)$, with $h_{-}^{(0+)}$ being the coefficient of the logarithmic term. The explicit forms of these functions are

$$
h_{-}^{(2)}(t, x)=k_{0}+k_{-}(t-x), \quad h_{-}^{(0)}(t, x)=\tilde{k}_{0}+k_{t} t+k_{x} x,
$$

with constant coefficients

$$
h_{-}^{(0+)}=2 k, \quad h_{-}^{(-4)}=-\ell^{6}(1+2 \gamma /(\ell \mu))\left(k_{x}+k_{t}\right)^{2} / 96 .
$$

where $k_{0}, k_{-}, \tilde{k}_{0}, k_{t}, k_{x}$, and $k$ are all arbitrary constants. However, periodicity in the angular coordinate $\phi \equiv x / \ell$ demands $k_{-}=k_{x}=0$.

Solutions with different chirality (i.e. with $H_{+} \neq 0$ at $c_{-}=0$ ) can also be found. For instance, one finds

$$
H_{+}(t, r, x)=h_{+}^{(2+)} r^{2} \log (r)+h_{+}^{(2)}(t, x) r^{2}+h_{+}^{(0)}(t, x)
$$

and $H_{-}=0$, with $h_{+}^{(2+)}$ being an arbitrary constant, and

$$
h_{+}^{(2)}(t, x)=k_{t} t+k_{x} x+k_{0}, \quad h_{+}^{(0)}(t, x)=\tilde{k}_{0}+k_{+}(t+x),
$$

where, again, $k_{t}, k_{x}, k_{0}, \tilde{k}_{0}$, and $k_{+}$are all arbitrary constants.

\subsection{Degeneracy and decoupling limit}

An interesting phenomenon occurs in the limit where the Cotton tensor disapears from the equations of motion (2.1). This corresponds to the limit $\mu \rightarrow \infty$ and $\gamma / \mu \rightarrow \infty$, keeping the ratio $m^{2} \equiv \mu^{2} / \gamma$ fixed. In this limit, central charges (2.8) read

$$
c_{ \pm}=\frac{3 \ell}{2 G}\left(\sigma+\frac{1}{2 m^{2} \ell^{2}}\right)
$$

and, therefore, the chiral point corresponds to $m^{2} \ell^{2} \sigma=-1 / 2$. At this point, the ansatz

$$
d s^{2}=d s_{\mathrm{eBTZ}}^{2}+H_{-}(r)(d t-\ell d \phi)^{2},
$$


with $H_{-}(r)$ depending only on $r$, solves the field equations for arbitrary $H_{-}(r)$. This is consistent with the fact that equation (3.4), when divided by $\mu^{3}$, is automatically satisfied for $m^{2} \ell^{2} \sigma=-1 / 2$ in the $\mu \rightarrow \infty$ limit. At this point of the parameter space, other solutions also exhibit special features. For instance, time-dependent solution (3.11) disapears as $N$ tends to zero in the limit $\mu \sim \gamma / \mu \rightarrow \infty$.

The type of degeneracy that (3.41) exhibits, is a common feature that higher-curvature theories present at special points of the moduli spaces where symmetry enhancement phenomena happen. This could also be a symptom indicating that the theory is not well defined in such limit. It would be interesting to understand this better.

\section{Conclusions}

In this paper, we have studied MMG theory, which is the extension of TMG proposed in Ref. [1] that solves the tension between bulk and boundary unitarity, a problem that theories like TMG and NMG have. Specifically, we studied MMG about $\mathrm{AdS}_{3}$ space at the chiral point, i.e. at the point of the parameter space where one of the central charges of the boundary theory vanishes. We considered both Brown-Henneaux [10] and Grumiller-Johansson [7] boundary conditions, which in the case of TMG at the chiral point lead to the definition of Chiral Gravity [5] and Log Gravity [9] theories, respectively.

We studied the theory at non-linear level; that is, we studied exact solutions to MMG field equation analytically, focusing our attention to solutions of MMG equations that are not Einstein manifolds. Then, we found two-parametric deformations of extremally rotating BTZ black holes. In particular, we found exact solutions that behave asymptotically as Log Gravity excitations. We computed the conserved charges of such configurations and showed that, despite the weakened $\mathrm{AdS}_{3}$ asymptotic, these Log deformations of BTZ black holes exhibit finite mass and finite angular momentum, which we compute.

We also found time-dependent deformations of extremal BTZ that depend on time linearly. These solutions do respect the stronger $\mathrm{AdS}_{3}$ boundary conditions, and it implies that at the chiral point solutions exist that behave asymptotically as Brown-Henneaux gravitons despite not being Einstein manifolds. Despite being time-dependent, these solutions have vanishing conserved charges. This opens the question about the contribution of such type of configurations to the partition function of the theory.

The general case we considered includes both Log Gravity and time-dependent deformations, representing two-parameter deformations of BTZ. This led us to show explicitly that Birkhoff theorem does not hold in this theory. We also investigate more general solutions that exist at the chiral point of MMG. Besides, the theory formulated at other points of the parameter space also exhibits interesting solutions of different types. For instance, it admits locally $\mathrm{AdS}_{2} \times \mathbb{R}$ geometries that are worth exploring.

This work was funded by CONICET Grant PIP 0595/13 and UBACyT 20020120100154BA (G.G.), and by Comisión Nacional de Ciencias y Tecnología through FONDECYT Grant 11121148 (Y.V.). G.G. thanks Pontificia Universidad Católica de Valparaíso for the hospitality. 


\section{References}

[1] E. Bergshoeff, O. Hohm, W. Merbis, A. Routh and P. Townsend, Class. Quant. Grav. 31 (2014) 145008.

[2] S. Deser, R. Jackiw and S. Templeton, Phys. Rev. Lett. 48 (1982) 975; Ann. Phys. 140 (1982) 372 .

[3] E. Bergshoeff, O. Hohm and P. Townsend, Phys. Rev. Lett. 102 (2009) 201301.

[4] A. Arvanitakis and P. Townsend, arXiv:1411.1970 [hep-th]].

[5] W. Li, W. Song and A. Strominger, JHEP 0804 (2008) 082.

[6] S. Carlip, S. Deser, A. Waldron and D. Wise, Class. Quant. Grav. 26 (2009) 075008; Phys. Lett. B 666 (2008) 272.

[7] D. Grumiller and N. Johansson, JHEP 0807 (2008) 134; Int. J. Mod. Phys. D 17 (2009) 2367. Erratum: S. Ertl et al., arXiv:0910.1706 [hep-th]].

[8] G. Giribet, M. Kleban and M. Porrati, JHEP 0810 (2008) 045.

[9] A. Maloney, W. Song and A. Strominger, Phys. Rev. D 81 (2010) 064007.

[10] J. Brown and M. Henneaux, Commun. Math. Phys. 104 (1986) 207.

[11] A. Garbarz, G. Giribet and Y. Vásquez, Phys. Rev. D 79 (2009) 044036.

[12] M. Bañados, C. Teitelboim and J. Zanelli, Phys. Rev. Lett. 69 (1992) 1849. M. Bañados, M. Henneaux, C. Teitelboim and J. Zanelli, Phys. Rev. D 48 (1993) 1506.

[13] G. Compère, S. de Buyl and S. Detournay, JHEP 1010 (2010) 042.

[14] O. Mišković and R. Olea, JHEP 0912 (2009) 046.

[15] A. Baykal, arXiv:1408.5232 [hep-th]].

[16] A. Arvanitakis, A. Routh and P. Townsend, [arXiv:1407.1264 [hep-th]].

[17] B. Tekin, Phys. Rev. D 90 (2014) 081701.

[18] A. Ayón-Beato and M. Hassaïne, Annals Phys. 317 (2005) 175; Phys. Rev. D 73 (2006) 104001. A. Ayón-Beato, G. Giribet and M. Hassaïne, JHEP 0905 (2009) 029.

[19] M. Alishahiha, M. Qaemmaqami, A. Naseh and A. Shirzad, arXiv:1409.6146 [hep-th]].

[20] S. Olmez, O. Sarioglu and B. Tekin, Class. Quant. Grav. 22 (2005) 4355.

[21] L. F. Abbott and S. Deser, Nucl. Phys. B 195 (1982) 76.

[22] S. Deser and B. Tekin, Phys. Rev. Lett. 89 (2002) 101101; Phys. Rev. D 67 (2003) 084009; Class. Quant. Grav. 19 (2002) L97; ibid. 20 (2003) L259.

[23] G. Giribet, A. Goya and M. Leston, Phys. Rev. D 84 (2011) 066003. 